\title{
Non-Markovian source term for particle production by a self-interacting scalar field in the large- $N$ approximation
}

\author{
Fuad M. Saradzhev ${ }^{1}$ \\ Institute of Physics, National Academy of Sciences of Azerbaijan, \\ H. Javid pr. 33, 370143 Baku, Azerbaijan
}

\begin{abstract}
The particle production in the self-interacting $N$-component complex scalar field theory is studied at large $N$. A non-Markovian source term that includes all higher order back-reaction and collision effects is derived. The kinetic amplitudes accounting for the change in the particle number density caused by collisions are obtained. It is shown that the production of particles is symmetric in the momentum space. The problem of renormalization is briefly discussed.
\end{abstract}

PACS: 11.10.z; 05.20.Dd; 11.10.Ef; 98.80.Cq

\section{Introduction}

In recent years an essential progress has been achieved in establishing a consistent kinetic description of particle production in quantum field theory from first principles. Investigations performed for QED with a constant [1] or time-dependent external electric field [2,3] have shown that a consistent field-theoretical approach leads to a kinetic equation with a modified source term, providing a nonMarkovian evolution of the distribution function.

Non-Markovian effects in collision terms have been studied in connection with relativistic heavy ion collisions [4], collective effects in nuclear matter [5], nuclear fragmentation [6], and the damping rates of giant dipole resonances [7]. The kinetic description of transport phenomena in QCD [8,9] and scalar field theories [10] has been also developed. A method to obtain quantum kinetic equations directly from the underlying quantum field theory implementing the Schwinger-Keldysh formalism and a dynamical renormalization group resummation has been presented in [11] . This method has been applied to different scalar and gauge theories, including the self-interacting $\varphi^{4}$-theory, yielding, however, no memory effects.

In the present paper, we aim to derive a non-Markovian quantum kinetic equation for a selfinteracting scalar field theory. We consider the model of a massive $N$-component complex scalar field in the large- $N$ approximation. Our purpose is to obtain a non-Markovian source term which incorporates all higher order, i.e. back-reaction and collisions effects.

In contrast with [11], in our paper we follow a kinetic approach introduced recently to study the decay of $C P$-odd metastable states and based originally on the evolution operator technique [12] . Herein we generalize this approach to include higher order effects.

\footnotetext{
1e-mail: fuad_saradzhev@hotmail.com
} 
Our plan is as follows. In Section 2 we introduce the model and perform the quantization of the scalar field fluctuations within the mean-field approximation. We construct the fluctuations Hamiltonian and identify its diagonalized part. In Section 3 we study at large $N$ the effects of the remaining nondiagonalized interactions in the particle production. We derive a quantum kinetic equation governing the production process and discuss its renormalization. We conclude with summary in Section 4.

\section{Quantization}

The Lagrangian density of our model is

$$
\mathcal{L}=\sum_{j=1}^{N}\left[\left(\partial_{\mu} \varphi_{j}^{\dagger}\right)\left(\partial^{\mu} \varphi_{j}\right)-m^{2} \varphi_{j}^{\dagger} \varphi_{j}\right]-V(\varphi),
$$

where $m$ is a bare mass. The model is defined in a finite volume $L^{3},-L / 2 \leq x_{i} \leq L / 2, i=1,2,3$, and the scalar field fulfils the periodic boundary conditions. In the continuum limit $\frac{1}{L^{3}} \sum_{\vec{k}}$, where the summation is over discrete momenta $\vec{k}=\frac{2 \pi}{L} \vec{n},(\vec{n})=\left(n_{1}, n_{2}, n_{3}\right)$, goes to $\int \frac{d^{3} \vec{k}}{(2 \pi)^{3}}$.

The potential is taken as

$$
V(\varphi)=\frac{\lambda}{2}\left(\sum_{j}^{N} \varphi_{j}^{\dagger} \varphi_{j}\right)^{2}, \quad \lambda>0,
$$

and includes self-interaction for each component as well as interaction of different components. From (1) we obtain the Klein-Gordon type equation of motion

$$
\left(\square+m^{2}\right) \varphi_{j}=J_{j}
$$

with the current

$$
J_{j} \equiv-\frac{\partial V}{\partial \varphi_{j}^{\dagger}}=-\lambda\left(\sum_{l=1}^{N} \varphi_{l}^{\dagger} \varphi_{l}\right) \varphi_{j}
$$

\subsection{Mean-field approximation}

Following the mean-field approximation, we usually decompose $\varphi_{j}(\vec{x}, t)$ into its vacuum mean value $\phi_{j}(t)=\left\langle\varphi_{j}(\vec{x}, t)\right\rangle$ and fluctuations $\chi_{j}$

$$
\varphi_{j}(\vec{x}, t)=\phi_{j}(t)+\chi_{j}(\vec{x}, t)
$$

with $\left\langle\chi_{j}(\vec{x}, t)\right\rangle=0$. The mean field is treated as a classical background field defined with respect to the in-vacuum $|0\rangle$ as

$$
\phi_{j}(t) \equiv \frac{1}{L^{3}} \int d^{3} x\left\langle 0\left|\varphi_{j}(\vec{x}, t)\right| 0\right\rangle,
$$

so that in the limit $t \rightarrow-\infty \phi_{j}(t) \rightarrow 0$, while the fluctuations are quantized and take place at all times.

The background $\phi$-fields are in general complex and contribute to the charge of the system. By a global $U(1)$ gauge transformation the phases of the $\phi$-fields can be put equal to zero at a fixed time. If the vanishing of the phases is compatible with equations of motion, it is possible to make them zero at all times as well. Otherwise, the background phases become dynamical variables coupled 
to the fluctuations in all orders. This results in new, non-diagonal terms in the Hamiltonian and therefore in new interactions.

To simplify the problem, it is convenient to choose the background neutral. Let us introduce the real fields

$$
\begin{aligned}
\phi_{1 j} & \equiv \frac{1}{2}\left(\phi_{j}+\phi_{j}^{\star}\right), \\
\phi_{2 j} & \equiv \frac{1}{2 i}\left(\phi_{j}-\phi_{j}^{\star}\right),
\end{aligned}
$$

and rewrite the decomposition (5) as

$$
\varphi_{j}(\vec{x}, t)=\phi_{1 j}(t)+\bar{\chi}_{j}(\vec{x}, t)
$$

where $\bar{\chi}_{j} \equiv \chi_{j}+i \phi_{2 j}$. The vacuum mean value of the "new" fluctuations is non-vanishing, $\left\langle\bar{\chi}_{j}\right\rangle=i \phi_{2 j}$.

Using Eq.(9) provides the following decomposition for the current

$$
J_{j}(\varphi)=J_{j}\left(\phi_{1}\right)-\mu_{0}^{2} \bar{\chi}_{j}-\mu_{j} \sum_{l=1}^{N} \mu_{l}\left(\bar{\chi}_{l}+\bar{\chi}_{l}^{\dagger}\right)+\bar{J}_{j}(\phi ; \bar{\chi}),
$$

where

$$
\begin{aligned}
\mu_{0}^{2} & \equiv \sum_{j=1}^{N} \mu_{j}^{2}, \\
\mu_{j} & \equiv \sqrt{\lambda} \phi_{1 j},
\end{aligned}
$$

while $\bar{J}_{j}(\phi ; \bar{\chi})$ includes terms of second and higher orders in fluctuations,

$$
\bar{J}_{j}(\phi ; \bar{\chi}) \equiv-\sqrt{\lambda} \mu_{j} \sum_{l=1}^{N} \bar{\chi}_{l}^{\dagger} \bar{\chi}_{l}-\sqrt{\lambda} \bar{\chi}_{j} \sum_{l=1}^{N} \mu_{l}\left(\bar{\chi}_{l}+\bar{\chi}_{l}^{\dagger}\right)+J_{j}(\bar{\chi})
$$

Substituting Eq.(9) also into (3) and taking the mean value $\langle\ldots\rangle$ yields the vacuum mean field equations

$$
\begin{aligned}
& \ddot{\phi}_{1 j}+\left(m^{2}+\mu_{0}^{2}\right) \phi_{1 j}=\operatorname{Re}\left\langle\bar{J}_{j}(\phi ; \bar{\chi})\right\rangle, \\
& \ddot{\phi}_{2 j}+\left(m^{2}+\mu_{0}^{2}\right) \phi_{2 j}=\operatorname{Im}\left\langle\bar{J}_{j}(\phi ; \bar{\chi})\right\rangle .
\end{aligned}
$$

As seen from (15), only if higher orders are neglected can the $\phi$-fields be taken neutral. Assuming that $\phi_{2 j}=0$ leads to the condition

$$
\operatorname{Im}\left\langle\bar{J}_{j}(\phi ; \bar{\chi})\right\rangle\left(\phi_{2}=0\right)=0
$$

which is not valid in general if the fluctuations are charged.

Eq.(3) in concert with Eqs.(14)-(15) provides also the equations of motion for the fluctuations. Introducing instead of $\bar{\chi}_{j}, \bar{\chi}_{j}^{\dagger}$ the hermitian fields

$$
\begin{gathered}
\bar{\chi}_{1 j} \equiv \frac{1}{\sqrt{2}}\left(\bar{\chi}_{j}+\bar{\chi}_{j}^{\dagger}\right), \\
\bar{\chi}_{2 j} \equiv \frac{1}{i \sqrt{2}}\left(\bar{\chi}_{j}-\bar{\chi}_{j}^{\dagger}\right),
\end{gathered}
$$

with $\left\langle\bar{\chi}_{1 j}\right\rangle=0,\left\langle\bar{\chi}_{2 j}\right\rangle=\phi_{2 j}$, yields

$$
\left(\square+m_{\alpha j}^{2}\right) \bar{\chi}_{\alpha j}=F_{\alpha j}
$$


$(\alpha=1,2)$, where

$$
\begin{gathered}
m_{1 j}^{2} \equiv m^{2}+\mu_{0}^{2}+2 \mu_{j}^{2} \\
m_{2 j}^{2} \equiv m^{2}+\mu_{0}^{2}
\end{gathered}
$$

are effective masses, and

$$
\begin{gathered}
F_{1 j} \equiv \bar{J}_{1 j}-\left\langle\bar{J}_{1 j}\right\rangle-2 \mu_{j} \sum_{\substack{l=1 \\
l \neq j}}^{N} \mu_{l} \bar{\chi}_{1 l}, \\
F_{2 j} \equiv \bar{J}_{2 j},
\end{gathered}
$$

the hermitian currents $\bar{J}_{\alpha j}$ being defined instead of $\bar{J}_{j}, \bar{J}_{j}^{\dagger}$ in the way analogous to (17)-(18).

Since the effective masses squared are always positive, the system evolves only in the non-tachyonic regime ( for the kinetic approach to the tachyonic regime, see [13] ). For $N=1$, the effective masses squared take the form $m_{1}^{2}=m^{2}+3 \lambda \phi_{1}^{2}, m_{2}^{2}=m^{2}+\lambda \phi_{1}^{2}$ in agreement with [14].

Rewriting (19) for the Fourier components $\bar{\chi}_{\alpha j}(\vec{k}, t), \alpha=1,2$, we obtain a Mathieu type equation

$$
\ddot{\bar{\chi}}_{\alpha j}(\vec{k}, t)+\omega_{\alpha j}^{2}(\vec{k}, t) \bar{\chi}_{\alpha j}(\vec{k}, t)=F_{\alpha j}(\vec{k}, t),
$$

where $F_{\alpha j}(\vec{k}, t)$ is the Fourier transform of $F_{\alpha j}(\vec{x}, t)$, and

$$
\omega_{\alpha j}^{2} \equiv \vec{k}^{2}+m_{\alpha j}^{2}
$$

are the time-dependent frequencies squared of the fluctuations. In the in-limit, $\omega_{\alpha j}$ become

$$
\lim _{t \rightarrow-\infty} \omega_{\alpha j}(\vec{k}, t) \equiv \omega^{0}(\vec{k})=\sqrt{(\vec{k})^{2}+m^{2}} .
$$

Eqs. (14),(15) and (24) are self-consistently coupled and include all higher-order effects. The vacuum mean fields modify the equations for the fluctuations via the time dependent frequencies, while the fluctuations react back on the $\phi$-fields via the source terms $\operatorname{Re}\left\langle\bar{J}_{j}\right\rangle$ and $\operatorname{Im}\left\langle\bar{J}_{j}\right\rangle$ in Eqs.(14),(15) and on the fluctuations themselves via the term $F_{\alpha j}(\vec{k}, t)$ in Eq.(24).

\subsection{Hamiltonian}

The fluctuations act on each other in two ways: directly, when higher orders are included, and via the $\phi$-fields. The potential (2) is decomposed as

$$
V(\varphi)=V\left(\phi_{1}\right)+\sqrt{2} \mu_{0}^{2} \sum_{j=1}^{N} \phi_{1 j} \bar{\chi}_{1 j}+\frac{\mu_{0}^{2}}{2} \sum_{j=1}^{N}\left(\bar{\chi}_{1 j}^{2}+\bar{\chi}_{2 j}^{2}\right)+\sum_{(j, l)=1}^{N} \mu_{j} \mu_{l} \bar{\chi}_{1 j} \bar{\chi}_{1 l}+\bar{V}(\phi ; \bar{\chi}),
$$

where $\bar{V}(\phi ; \bar{\chi})$ contains third and fourth orders in fluctuations,

$$
\bar{V}(\phi ; \bar{\chi})=-\sum_{j=1}^{N} \phi_{1 j}\left(J_{j}(\bar{\chi})+J_{j}^{\dagger}(\bar{\chi})\right)+V(\bar{\chi}) .
$$

With the decomposition (27), we deduce from (1) the Hamiltonian density governing the dynamics of the fluctuations

$$
\mathcal{H}_{\chi}=\frac{1}{2} \sum_{\alpha=1}^{2} \sum_{j=1}^{N}\left(\bar{\pi}_{\alpha j}^{2}+\left(\vec{\nabla} \bar{\chi}_{\alpha j}\right)^{2}+m_{\alpha j}^{2} \bar{\chi}_{\alpha j}^{2}\right)+\sum_{\substack{j, l)=1 \\ j \neq l}}^{N} \mu_{j} \mu_{l} \bar{\chi}_{1 j} \bar{\chi}_{1 l}+\overline{\mathcal{H}}_{\chi}
$$


where $\bar{\pi}_{\alpha j}$ are the momenta canonically conjugate to $\bar{\chi}_{\alpha j}$, and

$$
\overline{\mathcal{H}}_{\chi} \equiv \bar{V}\left(\phi_{1} ; \bar{\chi}\right)+\sum_{j=1}^{N}\left\langle\bar{J}_{1 j}\right\rangle \bar{\chi}_{1 j}
$$

The expression (29) is not diagonal even in the second order in fluctuations. The non-diagonal terms include interactions of fields of different values of $j$. form

In terms of the Fourier components $\bar{\chi}_{\alpha j}(\vec{k}, t), \bar{\pi}_{\alpha j}(\vec{k}, t)$, the fluctuations Hamiltonian takes the

$$
H_{\chi}=\int d^{3} x \mathcal{H}_{\chi}=\sum_{j=1}^{N} H_{j}+\sum_{\substack{(j, l)=1 \\ j \neq l}}^{N} H_{j l}+L^{3 / 2} \overline{\mathcal{H}}_{\chi}(\vec{k}=0, t),
$$

where $\overline{\mathcal{H}}_{\chi}(\vec{k}, t)$ is the Fourier transform of the potential $\overline{\mathcal{H}}_{\chi}(\vec{x}, t)$, and

$$
\begin{gathered}
H_{j} \equiv \frac{1}{2} \sum_{\alpha=1}^{2} \sum_{\vec{k}}\left(\bar{\pi}_{\alpha j}^{\dagger}(\vec{k}, t) \bar{\pi}_{\alpha j}(\vec{k}, t)+\omega_{\alpha j}^{2}(\vec{k}, t) \bar{\chi}_{\alpha j}^{\dagger}(\vec{k}, t) \bar{\chi}_{\alpha j}(\vec{k}, t)\right), \\
H_{j l} \equiv \mu_{j} \mu_{l} \sum_{\vec{k}} \bar{\chi}_{1 j}(\vec{k}, t) \bar{\chi}_{1 l}^{\dagger}(\vec{k}, t) .
\end{gathered}
$$

The Hamiltonian equations of motion are

$$
\begin{aligned}
& \dot{\bar{\chi}}_{\alpha j}(\vec{k}, t)=\bar{\pi}_{\alpha j}^{\dagger}(\vec{k}, t), \\
& \dot{\bar{\pi}}_{\alpha j}(\vec{k}, t)=-\omega_{\alpha j}^{2}(\vec{k}, t) \bar{\chi}_{\alpha j}^{\dagger}(\vec{k}, t)+F_{\alpha j}^{\dagger}(\vec{k}, t),
\end{aligned}
$$

in agreement with (24).

By the transition to the new fields (17)-(18), we have achieved a partial diagonalization of the Hamiltonian in the second order in fluctuations. Its complete diagonalization, including all higher order, i.e. interaction terms, is a very complicated problem. However, there are different approximation schemes which allow one to diagonalize a "physically" important part of the interaction, while the remaining part is treated as a small perturbation.

Let us assume that, by using one of these schemes, we succeeded in diagonalising some of the interaction terms in our model. We assume next that a new diagonalized part of the Hamiltonian looks like the one in Eq.(32), the frequencies of fluctuations being only modified. Namely, $\omega_{\alpha j}^{2}(\vec{k}, t)$ is replaced by

$$
\tilde{\omega}_{\alpha j}^{2}(\vec{k}, t) \equiv \omega_{\alpha j}^{2}(\vec{k}, t)+M_{\alpha j}^{2}(\vec{k}, t),
$$

where $M_{\alpha j}^{2}(\vec{k}, t)$ represents the higher order effects contribution. The explicit expression for $M_{\alpha j}^{2}(\vec{k}, t)$ depends on the approximation scheme under consideration. In the next section, we will calculate $M_{\alpha j}^{2}(\vec{k}, t)$ in the large- $N$ approximation.

For the Hermitian fluctuations, we introduce then the annihilation and creation operators $d_{\alpha j}(\vec{k}, t)$ and $d_{\alpha j}^{\dagger}(\vec{k}, t)$ in a standard way by

$$
\bar{\chi}_{\alpha j}(\vec{k}, t)=\Gamma_{\alpha j}(\vec{k}, t) d_{\alpha j}(\vec{k}, t)+\Gamma_{\alpha j}^{\star}(\vec{k}, t) d_{\alpha j}^{\dagger}(-\vec{k}, t)
$$

and

$$
\bar{\pi}_{\alpha j}(\vec{k}, t)=-i \tilde{\omega}_{\alpha j}(\vec{k}, t)\left[\Gamma_{\alpha j}(\vec{k}, t) d_{\alpha j}(-\vec{k}, t)-\Gamma_{\alpha j}^{\star}(\vec{k}, t) d_{\alpha j}^{\dagger}(\vec{k}, t)\right]
$$


where

$$
\Gamma_{\alpha j}(\vec{k}, t)=\frac{1}{\sqrt{2 \tilde{\omega}_{\alpha j}(\vec{k}, t)}} \exp \left\{-i \Theta_{\alpha j}\left(\tilde{\omega}_{\alpha j}, t\right)\right\}
$$

and $\Theta_{\alpha j}\left(\tilde{\omega}_{\alpha j}, t\right)$ are arbitrary phases. In the case when higher order effects are omitted, i.e. $M_{\alpha j}^{2}=0$, the phases $\Theta_{\alpha j}\left(\omega_{\alpha j}, t\right)$ take the form $\omega_{k}^{(0)} t$ in the in-limit, and the operators $\tilde{\chi}_{\alpha j}(\vec{k}, t), \tilde{\pi}_{\alpha j}(\vec{k}, t)$ can be connected with the corresponding in-field ones by making use of the evolution operator [12].

The ansatz (37) ( and (38) ) has the same structure as the free field theory one except the frequencies $\tilde{\omega}_{\alpha j}(\vec{k}, t)$ replace $\omega^{(0)}(\vec{k})$, and the time-independent annihilation and creation operators are replaced here by the time-dependent ones $d_{\alpha j}(\vec{k}, t), d_{\alpha j}^{\dagger}(\vec{k}, t)$. The Hamiltonians $H_{j}$ become

$$
H_{j}=\frac{1}{2} \sum_{\alpha=1}^{2} \sum_{\vec{k}} \tilde{\omega}_{\alpha j}(\vec{k}, t)\left(d_{\alpha j}^{\dagger}(\vec{k}, t) d_{\alpha j}(\vec{k}, t)+d_{\alpha j}(\vec{k}, t) d_{\alpha j}^{\dagger}(\vec{k}, t)\right) .
$$

\section{Kinetic equations}

There are $2 N$ types of neutral particles in our model which are related to $2 N$ types of the fluctuations $\bar{\chi}_{\alpha j}$. The numbers of these particles of momentum $\vec{k}$ at time $t$ are given by the occupation number densities

$$
\mathcal{N}_{\alpha j}(\vec{k}, t) \equiv\left\langle 0\left|d_{\alpha j}^{\dagger}(\vec{k}, t) d_{\alpha j}(\vec{k}, t)\right| 0\right\rangle .
$$

In the limit $t \rightarrow-\infty$, these densities vanish because the initial state is assumed to be "empty", i.e. without particles.

In general, $\mathcal{N}_{\alpha j}(\vec{k}, t)$ and $\mathcal{N}_{\alpha j}(-\vec{k}, t)$ are not equal. Therefore it is convenient to introduce

$$
\mathcal{N}_{\alpha j, \pm}(\vec{k}, t) \equiv \frac{1}{2}\left(\mathcal{N}_{\alpha j}(\vec{k}, t) \pm \mathcal{N}_{\alpha j}(-\vec{k}, t)\right)
$$

where $\mathcal{N}_{\alpha j,+}(\vec{k}, t)$ is the particle number averaged over the directions $\vec{k}$ and $(-\vec{k})$, while $\mathcal{N}_{\alpha j,-}(\vec{k}, t)$ measures the degree of asymmetry.

We consider first the time evolution of $\mathcal{N}_{\alpha j,-}(\vec{k}, t)$. Using the relations

$$
d_{\alpha j}(\vec{k}, t)=\frac{1}{2 \Gamma_{\alpha j}(\vec{k}, t)}\left(\bar{\chi}_{\alpha j}(\vec{k}, t)+\frac{i}{\tilde{\omega}_{\alpha j}(\vec{k}, t)} \bar{\pi}_{\alpha j}^{\dagger}(\vec{k}, t)\right)
$$

and the Hamiltonian equations of motion (34) - (35), we obtain

$$
\dot{\mathcal{N}}_{\alpha j,-}(\vec{k}, t)=-\operatorname{Im}\left\langle 0\left|\bar{\chi}_{\alpha j}^{\dagger}(\vec{k}, t) F_{\alpha j}(\vec{k}, t)\right| 0\right\rangle .
$$

If we omit higher-order effects and take $\bar{J}_{\alpha j}=0$, then

$$
\dot{\mathcal{N}}_{\alpha j,-}(\vec{k}, t)=2 \mu_{j} \delta_{\alpha 1} \sum_{\substack{l=1 \\ l \neq j}}^{N} \mu_{l} \operatorname{Im}\left\langle 0\left|\bar{\chi}_{1 j}^{\dagger}(\vec{k}, t) \bar{\chi}_{1 l}(\vec{k}, t)\right| 0\right\rangle,
$$

i.e. the time evolution of the asymmetry in the production of particles by the field of a fixed value of $j$ is determined by its interaction with other fields. For $N=1$, the density $\mathcal{N}_{\alpha 1,-}(\vec{k}, t)$ is conserved, $\dot{\mathcal{N}}_{\alpha 1,-}(\vec{k}, t)=0$, so if we take $\mathcal{N}_{\alpha 1,-}\left(\vec{k}, t_{0}\right)=0$, where $t_{0}$ is a moment of time at which the particle production starts, then the asymmetry does not appear at any time $t$ later. 
Taking next the time derivative of $\mathcal{N}_{\alpha j,+}(\vec{k}, t)$, yields the evolution equation

$$
\dot{\mathcal{N}}_{\alpha j,+}(\vec{k}, t)=\frac{\dot{\tilde{\omega}}_{\alpha j}}{\tilde{\omega}_{\alpha j}} \operatorname{Re}\left[e^{-2 i \Theta_{\alpha j}} C_{\alpha j}(\vec{k}, t)\right]+\Delta_{\alpha j}^{(1)}(\vec{k}, t),
$$

where we have defined the time-dependent pair correlation functions

$$
C_{\alpha j}(\vec{k}, t) \equiv\left\langle 0\left|d_{\alpha j}(-\vec{k}, t) d_{\alpha j}(\vec{k}, t)\right| 0\right\rangle
$$

and

$$
\Delta_{\alpha j}^{(1)} \equiv \frac{1}{\tilde{\omega}_{\alpha j}} \operatorname{Re}\left\langle 0\left|\bar{\pi}_{\alpha j}(\vec{k}, t) \tilde{F}_{\alpha j}(\vec{k}, t)\right| 0\right\rangle
$$

with

$$
\tilde{F}_{\alpha j}(\vec{k}, t) \equiv F_{\alpha j}(\vec{k}, t)+M_{\alpha j}^{2}(\vec{k}, t) \bar{\chi}_{\alpha j}(\vec{k}, t) .
$$

The pair correlation functions $C_{\alpha j}(\vec{k}, t)$ can be shown to obey the equations

$$
\dot{C}_{\alpha j}(\vec{k}, t)-2 i\left(\dot{\Theta}_{\alpha j}-\tilde{\omega}_{\alpha j}\right) C_{\alpha j}(\vec{k}, t)=\left[\frac{\dot{\tilde{\omega}}_{\alpha j}}{2 \tilde{\omega}_{\alpha j}}\left(1+2 \mathcal{N}_{\alpha j,+}(\vec{k}, t)\right)+\left(-\Delta_{\alpha j}^{(1)}(\vec{k}, t)+i \Delta_{\alpha j}^{(2)}(\vec{k}, t)\right)\right] e^{2 i \Theta_{\alpha j}},
$$

where

$$
\Delta_{\alpha j}^{(2)}(\vec{k}, t) \equiv \operatorname{Re}\left\langle 0\left|\bar{\chi}_{\alpha j}^{\dagger}(\vec{k}, t) \tilde{F}_{\alpha j}(\vec{k}, t)\right| 0\right\rangle .
$$

As seen from Eqs.(46) and (50), the higher order effects contribute to the evolution equations via the frequencies $\tilde{\omega}_{\alpha j}$ and via the functions $\Delta_{\alpha j}^{(1)}(\vec{k}, t)$ and $\Delta_{\alpha j}^{(2)}(\vec{k}, t)$. These functions include higher order correlation functions, namely, the vacuum expectation values of the products of three and four field operators.

\subsection{Large- $N$ approximation}

To calculate $\Delta_{\alpha j}^{(1)}(\vec{k}, t), \Delta_{\alpha j}^{(2)}(\vec{k}, t)$, we use the large- $N$ approximation [15-18]. At large $N$, the theory , which consists of an infinite number of coupled correlation function equations, reduces to the solution of coupled equations for the one- and two-point functions. For simplicity, we consider the case of spatially homogeneous vacuum configurations and assume that $\left\langle 0\left|\bar{\chi}_{2 j}(\vec{x}, t)\right| 0\right\rangle$ is a function only of time, then

$$
\left\langle 0\left|\bar{\chi}_{2 j}(\vec{x}, t)\right| 0\right\rangle=\phi_{2 j} .
$$

The basic points of the large $N$-approximation are as follows. (i) The equal time two-point correlation functions are proportional to $\delta_{\alpha, \beta}(\alpha, \beta=\overline{1,2})$ and $\delta_{j l}(j, l=\overline{1, N})$ unless the vacuum expectation values of $\bar{\chi}_{\alpha j}$ are non-zero. Assuming translational invariance of the vacuum state $|0\rangle$, we define the correlation functions

$$
\begin{gathered}
\left\langle 0\left|\bar{\chi}_{1 j}(\vec{x}, t) \bar{\chi}_{1 l}(\vec{y}, t)\right| 0\right\rangle=\delta_{j l} G_{1 j}(\vec{x}-\vec{y} ; t), \\
\left\langle 0\left|\bar{\chi}_{2 j}(\vec{x}, t) \bar{\chi}_{2 l}(\vec{y}, t)\right| 0\right\rangle=\phi_{2 j} \phi_{2 l}+\delta_{j l} G_{2 j}(\vec{x}-\vec{y} ; t),
\end{gathered}
$$

and

$$
\begin{gathered}
\left\langle 0\left|\bar{\pi}_{1 j}(\vec{x}, t) \bar{\chi}_{1 l}(\vec{y}, t)\right| 0\right\rangle=\delta_{j l} D_{1 j}(\vec{x}-\vec{y} ; t), \\
\left\langle 0\left|\bar{\pi}_{2 j}(\vec{x}, t) \bar{\chi}_{2 l}(\vec{y}, t)\right| 0\right\rangle=\dot{\phi}_{2 j} \phi_{2 l}+\delta_{j l} D_{2 j}(\vec{x}-\vec{y} ; t),
\end{gathered}
$$

while

$$
\left\langle 0\left|\bar{\chi}_{1 j}(\vec{x}, t) \bar{\chi}_{2 l}(\vec{y}, t)\right| 0\right\rangle=\left\langle 0\left|\bar{\pi}_{1 j}(\vec{x}, t) \bar{\chi}_{2 l}(\vec{y}, t)\right| 0\right\rangle=\left\langle 0\left|\bar{\pi}_{2 j}(\vec{x}, t) \bar{\chi}_{1 l}(\vec{y}, t)\right| 0\right\rangle=0 .
$$


(ii) The vacuum values of all fields are the same, i.e. $\phi_{1 j}=\phi_{1}$ and $\phi_{2 j}=\phi_{2}$ for all values of $j$. As a consequence, $\mu_{0}^{2}=\bar{\lambda} \phi_{1}^{2}$ where $\bar{\lambda} \equiv \lambda N$, and the effective masses coincide, $m_{1 j}^{2}=m_{2 j}^{2} \equiv m_{j}^{2}=$ $m^{2}+\bar{\lambda} \phi_{1}^{2}$. The correlation functions $G_{\alpha j}, D_{\alpha j}$ as well do not depend on the particular value of $j$. However, in what follows we keep the index $j$ on all correlation functions, vacuum mean values of currents and etc, except the $\phi$-fields.

(iii) The higher order correlation functions are factorized into products of two-point correlation functions and vacuum fields. For example,

$$
\begin{gathered}
\left\langle 0\left|\bar{\chi}_{2 j}(\vec{x}, t) \bar{\chi}_{1 l}(\vec{x}, t) \bar{\chi}_{1 l}(\vec{x}, t)\right| 0\right\rangle=\phi_{2} G_{1 l}(0 ; t) \\
\left\langle 0\left|\bar{\chi}_{2 j}(\vec{x}, t) \bar{\chi}_{2 l}(\vec{x}, t) \bar{\chi}_{2 l}(\vec{x}, t)\right| 0\right\rangle=\phi_{2}\left[G_{2 l}(0 ; t)+2 \delta_{j l} G_{2 j}(0 ; t)+3 \phi_{2}^{2}\right] .
\end{gathered}
$$

With these points it is straightforward to calculate $\Delta_{\alpha j}^{(1)}(\vec{k}, t)$ and $\Delta_{\alpha j}^{(2)}(\vec{k}, t)$. We start, however, with the vacuum mean value of the hermitian currents $\bar{J}_{\alpha j}(\vec{x}, t)$. Using (13) yields for these currents the following expressions

$$
\begin{gathered}
\bar{J}_{1 j}=-\sqrt{2 \lambda} \bar{\chi}_{1 j} \sum_{l=1}^{N} \mu_{l} \bar{\chi}_{1 l}-\frac{1}{2}\left(\sqrt{2 \lambda} \mu_{j}+\lambda \bar{\chi}_{1 j}\right) \sum_{l=1}^{N}\left(\bar{\chi}_{1 l}^{2}+\bar{\chi}_{2 l}^{2}\right), \\
\bar{J}_{2 j}=-\sqrt{2 \lambda} \bar{\chi}_{2 j} \sum_{l=1}^{N} \mu_{l} \bar{\chi}_{1 l}-\frac{\lambda}{2} \bar{\chi}_{2 j} \sum_{l=1}^{N}\left(\bar{\chi}_{1 l}^{2}+\bar{\chi}_{2 l}^{2}\right) .
\end{gathered}
$$

Using next the factorization (iii), we obtain

$$
\begin{gathered}
\left\langle\bar{J}_{1 j}\right\rangle=-\frac{\bar{\lambda}}{\sqrt{2}} \Omega_{j}^{2} \phi_{1}, \\
\left\langle\bar{J}_{2 j}\right\rangle=-\frac{\bar{\lambda}}{2}\left[\Omega_{j}^{2}+3 \phi_{2}^{2}\right] \phi_{2},
\end{gathered}
$$

where

$$
\Omega_{j}^{2} \equiv G_{1 j}(0 ; t)+G_{2 j}(0 ; t)=\frac{1}{L^{3}} \sum_{\alpha=1}^{2} \sum_{\vec{k}}\left\langle 0\left|\bar{\chi}_{\alpha j}^{\dagger}(\vec{k}, t) \bar{\chi}_{\alpha j}(\vec{k}, t)\right| 0\right\rangle .
$$

As seen from Eq.(63), $\left\langle\bar{J}_{2 j}\right\rangle=0$ for $\phi_{2}=0$, i.e. the condition (16) is fulfilled. We come therefore to the important conclusion: in the large- $N$ approximation the $\phi$-fields can be taken neutral at all times. Henceforth we put $\phi_{2}=0$.

Substituting Eq.(62) into Eq.(14), we rewrite the vacuum mean field equation as

$$
\ddot{\phi}_{1 j}+\left(m_{j}^{2}+\frac{\bar{\lambda}}{2} \Omega_{j}^{2}\right) \phi_{1 j}=0
$$

so that the higher order effects contribution to the effective masses is

$$
M_{j}^{2}=\frac{\bar{\lambda}}{2} \Omega_{j}^{2}
$$

We turn now to the calculation of $\Delta_{\alpha j}^{(1)}(\vec{k}, t)$ and $\Delta_{\alpha j}^{(2)}(\vec{k}, t)$. In the large- $N$ approximation, due to the point $(i)$ the interaction of components with different values of $j$ does not contribute to the 
vacuum expectation values, and $\Delta_{\alpha j}^{(1)}(\vec{k}, t)$ and $\Delta_{\alpha j}^{(2)}(\vec{k}, t)$ take the form

$$
\begin{aligned}
& \Delta_{\alpha j}^{(1)}(\vec{k}, t)=\frac{1}{\tilde{\omega}_{\alpha j}} \operatorname{Re}\left\langle 0\left|\bar{\pi}_{\alpha j}(\vec{k}, t) \bar{J}_{\alpha j}(\vec{k}, t)\right| 0\right\rangle+\frac{\bar{\lambda}}{2 \tilde{\omega}_{\alpha j}} \Omega_{j}^{2} \cdot \operatorname{Re} D_{\alpha j}(\vec{k}, t), \\
& \Delta_{\alpha j}^{(2)}(\vec{k}, t)=\operatorname{Re}\left\langle 0\left|\bar{\chi}_{\alpha j}^{\dagger}(\vec{k}, t) \bar{J}_{\alpha j}(\vec{k}, t)\right| 0\right\rangle+\frac{\bar{\lambda}}{2} \Omega_{j}^{2} \cdot G_{\alpha j}(\vec{k}, t),
\end{aligned}
$$

where $D_{\alpha j}(\vec{k}, t)$ and $G_{\alpha j}(\vec{k}, t)$ are the Fourier transforms of $D_{\alpha j}(\vec{x}-\vec{y} ; t)$ and $G_{\alpha j}(\vec{x}-\vec{y} ; t)$, respectively.

Evaluating next the self-interaction contribution by making use of (iii), we obtain

$$
\begin{aligned}
\operatorname{Re}\left\langle 0\left|\bar{\pi}_{\alpha j}(\vec{k}, t) \bar{J}_{\alpha j}(\vec{k}, t)\right| 0\right\rangle & =-\frac{\bar{\lambda}}{2} \Omega_{j}^{2} \cdot \operatorname{Re} D_{\alpha j}(\vec{k}, t), \\
\operatorname{Re}\left\langle 0\left|\bar{\chi}_{\alpha j}^{\dagger}(\vec{k}, t) \bar{J}_{\alpha j}(\vec{k}, t)\right| 0\right\rangle & =-\frac{\bar{\lambda}}{2} \Omega_{j}^{2} \cdot G_{\alpha j}(\vec{k}, t),
\end{aligned}
$$

leading finally to

$$
\Delta_{\alpha j}^{(1)}(\vec{k}, t)=\Delta_{\alpha j}^{(2)}(\vec{k}, t)=0 .
$$

In the same way we calculate the vacuum expectation value in the r.h.s. of (44) and obtain that

$$
\dot{\mathcal{N}}_{\alpha j,-}(\vec{k}, t)=0
$$

i.e. in the large- $N$ approximation the particle production is symmetric in the momentum space for all times.

With $\Delta_{\alpha j}^{(1)}=\Delta_{\alpha j}^{(2)}=0$, Eq.(50) is solved by

$$
C_{\alpha j}(\vec{k}, t)=e^{2 i \Theta_{\alpha j}} \int_{t_{0}}^{t} d t^{\prime} \frac{\dot{\tilde{\omega}}_{\alpha j}}{2 \tilde{\omega}_{\alpha j}}\left(\vec{k}, t^{\prime}\right)\left(1+2 \mathcal{N}_{\alpha j,+}\left(\vec{k}, t^{\prime}\right)\right) \cdot e^{2 i\left(\tilde{\Theta}_{\alpha j}\left(\vec{k}, t^{\prime}\right)-\tilde{\Theta}_{\alpha j}(\vec{k}, t)\right)},
$$

where

$$
\tilde{\Theta}_{\alpha j}(\vec{k}, t) \equiv \int_{t_{0}}^{t} d t^{\prime} \tilde{\omega}_{\alpha j}\left(\vec{k}, t^{\prime}\right)
$$

Substituting (73) into (46), we obtain the following closed equation for $\mathcal{N}_{\alpha j,+}(\vec{k}, t)$ :

$$
\dot{\mathcal{N}}_{\alpha j,+}(\vec{k}, t)=\frac{1}{2} \tilde{W}_{\alpha j}(\vec{k}, t) \int_{t_{0}}^{t} d t^{\prime} \tilde{W}_{\alpha j}\left(\vec{k}, t^{\prime}\right) \cdot\left(1+2 \mathcal{N}_{\alpha j,+}\left(\vec{k}, t^{\prime}\right)\right) \cos \left[\tilde{x}_{\alpha j}\left(\vec{k} ; t^{\prime}, t\right)\right],
$$

where

$$
\tilde{W}_{\alpha j} \equiv \frac{\dot{\tilde{\omega}}_{\alpha j}}{\tilde{\omega}_{\alpha j}}
$$

are kinetic amplitudes, while

$$
\tilde{x}_{\alpha j}\left(\vec{k} ; t^{\prime}, t\right) \equiv 2\left[\tilde{\Theta}_{\alpha j}\left(\vec{k}, t^{\prime}\right)-\tilde{\Theta}_{\alpha j}(\vec{k}, t)\right]
$$

is the phase difference.

Eq.(75) is a complete quantum kinetic equation in the large- $N$ approximation with all higher order effects included. It determines the time evolution of the number of particles of a fixed $j$ and a fixed momentum $\vec{k}$. 
The background $\phi$-fields are given by solution of the non-linear equation (65), while $\Omega_{j}^{2}$ must be determined self-consistently from

$$
\Omega_{j}^{2}=\frac{1}{L^{3}} \sum_{\alpha=1}^{2} \sum_{\vec{k}} \frac{1}{2 \tilde{\omega}_{\alpha j}}\left[\left(1+2 \mathcal{N}_{\alpha j,+}(\vec{k}, t)\right)+2 R_{\alpha j}(\vec{k}, t)\right]
$$

with

$$
R_{\alpha j}(\vec{k}, t)=\frac{1}{2} \int_{t_{0}}^{t} d t^{\prime} \tilde{W}_{\alpha j}\left(\vec{k}, t^{\prime}\right)\left(1+2 \mathcal{N}_{\alpha j,+}\left(\vec{k}, t^{\prime}\right)\right) \cos \left[\tilde{x}_{\alpha j}\left(\vec{k} ; t^{\prime}, t\right)\right] .
$$

We have therefore a system of three coupled equations.

The kinetic equation (75) has the following important features: (i) The source term, i.e. its r.h.s. is non-Markovian. Therefore calculating the particle density at any given instant requires a complete knowledge of the history of the production process. In addition, the integrand is a non-local function of time, which apparent in the coherent phase oscillation term $\cos \left[\tilde{x}_{\alpha j}\left(\vec{k} ; t^{\prime}, t\right)\right]$; (ii) The background $\phi$-fields do not contribute to the kinetic equation directly, but only via the frequencies of the quantum fluctuations, as evident in Eqs.(76) and (77); (iii) The production rate is affected by the produced particles' statistics, as seen in the statistical factor $\left(1+2 \mathcal{N}_{\alpha j,+}(\vec{k}, t)\right)$. In the case of fermions, this factor would be $\left(1-2 \mathcal{N}_{\alpha j,+}(\vec{k}, t)\right)$.

The kinetic amplitudes $\tilde{W}_{\alpha j}(\vec{k}, t)$ are decomposed as

$$
\tilde{W}_{\alpha j}=W_{\alpha j}+W_{\alpha j}^{\Omega},
$$

where

$$
W_{\alpha j} \equiv \frac{\dot{\omega}_{\alpha j}}{\omega_{\alpha j}}
$$

and

$$
W_{\alpha j}^{\Omega} \equiv \frac{\bar{\lambda}}{2} \frac{\Omega_{j}}{\omega_{\alpha j}^{2}}\left(\dot{\Omega}_{j}-\Omega_{j} W_{\alpha j}\right)\left(1+\frac{\bar{\lambda}}{2} \frac{\Omega_{j}^{2}}{\omega_{\alpha j}^{2}}\right)^{-1} .
$$

The amplitudes $W_{\alpha j}(\vec{k}, t)$ account for the increase in the particle number caused by the transition of energy from the background $\phi$-fields to fluctuations. As soon as the energy transition stops, the amplitudes $W_{\alpha j}(\vec{k}, t)$ vanish. If, for example, the time evolution of the $\phi$-fields starts at $t=t_{0}$ and ends at $t=t_{1}$, then $W_{\alpha j}(\vec{k}, t)=0$ for all $t>t_{1}$.

In contrast with $W_{\alpha j}(\vec{k}, t)$, the new amplitudes $W_{\alpha j}^{\Omega}(\vec{k}, t)$ account for the change in the particle number density caused by the interaction of the particles produced, i.e. by their collisions, and do not vanish when the particle production by the background fields stops. The fluctuations interact with each other in two ways, directly and via the $\phi$-fields. For $t>t_{1}$, the time-dependence of the particle number density is only governed by collisions.

Collisions also modify the expression for the dynamical phase. If collision effects are omitted, $\tilde{\Theta}_{\alpha j}$ reduces to the adiabatic phase

$$
\Theta_{\alpha j}^{a d}(\vec{k}, t) \equiv \int_{t_{0}}^{t} d t^{\prime} \omega_{\alpha j}\left(\vec{k}, t^{\prime}\right)
$$

that takes the form $\omega_{k}^{(0)}\left(t-t_{0}\right)$ in the in-limit. With collisions taken into account, the dynamical phase is no longer the adiabatic one and the connection with the in-picture no longer exists.

The non-Markovian kinetic equation in the collisionless limit can be simply obtained from (75) by replacing $\tilde{W}_{\alpha j}$ with $W_{\alpha j}$ and $\tilde{\Theta}_{\alpha j}$ with $\Theta_{\alpha j}^{a d}$. The kinetic equations in both cases, i.e. with and without collisions, have therefore the same non-Markovian structure. This is a result of the large- $N$ approximation. However, collisions introduce non-Markovian effects in addition to those already present in the case without collisions. Even in the "collision" regime for $t>t_{1}$, the production rate is affected by the background fields induced particle production process from $t_{0}$ to $t_{1}$. 


\subsection{Renormalization}

All the divergences in our approach come from the expression for $\tilde{\omega}_{\alpha j}(t)$ :

$$
\tilde{\omega}_{\alpha j}^{2}=\vec{k}^{2}+m_{\alpha j}^{2}(t)+\frac{\bar{\lambda}}{2} \Omega_{j}^{2}(t)
$$

the sum over $\vec{k}$ in the definition of $\Omega_{j}^{2}[$ Eq.(78)] having both quadratic and logarithmic divergences which have to be absorbed by mass and coupling constant renormalization [18] . The subtraction of $\Omega_{j}^{2}\left(t_{0}\right)$ from $\Omega_{j}^{2}(t)$ removes the quadratic divergence. With $\mathcal{N}_{\alpha j,+}\left(\vec{k}, t_{0}\right)=R_{\alpha j}\left(\vec{k}, t_{0}\right)=0$, we rewrite $\tilde{\omega}_{\alpha j}^{2}(t)$ as

$$
\tilde{\omega}_{\alpha j}^{2}(t)=\omega_{R}^{2}+S_{j}(t),
$$

where

$$
\omega_{R}^{2} \equiv \vec{k}^{2}+m_{R}^{2}
$$

$m_{R}^{2}$ being the renormalized mass squared,

$$
m_{R}^{2} \equiv m^{2}+\bar{\lambda} \phi_{1}^{2}\left(t_{0}\right)+\frac{\bar{\lambda}}{2} \Omega_{j}^{2}\left(t_{0}\right),
$$

and

$$
S_{j}(t) \equiv \bar{\lambda}\left[\phi_{1}^{2}(t)-\phi_{1}^{2}\left(t_{0}\right)\right]+\frac{\bar{\lambda}}{2}\left[\Omega_{j}^{2}(t)-\Omega_{j}^{2}\left(t_{0}\right)\right]
$$

$S_{j}\left(t_{0}\right)=0$.

The remaining logarithmic divergence is removed by the coupling constant renormalization. The bare coupling constant and the renormalized one are related by the geometric series

$$
\bar{\lambda}=\frac{\lambda_{R}}{1-\lambda_{R} \delta \lambda}
$$

where

$$
\delta \lambda \equiv \frac{1}{L^{3}} \sum_{\vec{k}} \frac{1}{4 \omega_{R}^{3}(\vec{k})} .
$$

Multiplying both sides of $(88)$ by $\left(1-\lambda_{R} \delta \lambda\right)$, we obtain the finite equation

$$
S_{j}(t)=\lambda_{R}\left[\phi_{1}^{2}(t)-\phi_{1}^{2}\left(t_{0}\right)\right]+\frac{\lambda_{R}}{2}\left[\Omega_{j}^{2}(t)-\Omega_{j}^{2}\left(t_{0}\right)+2 \delta \lambda \cdot S_{j}(t)\right] .
$$

If we expand $\Omega_{j}^{2}(t)$ and $S_{j}(t)$ in a power series in $\lambda_{R}$,

$$
\begin{gathered}
\Omega_{j}^{2}(t)=\Omega_{j(0)}^{2}(t)+\sum_{n=1}^{\infty} \lambda_{R}^{n} \cdot \Omega_{j(n)}^{2}(t), \\
S_{j}(t)=\sum_{n=1}^{\infty} \lambda_{R}^{n} \cdot S_{j(n)}(t)
\end{gathered}
$$

and choose the initial conditions for $\Omega_{j}^{2}(t)$ as

$$
\begin{gathered}
\Omega_{j}^{2}\left(t_{0}\right)=\Omega_{j(0)}^{2}\left(t_{0}\right), \\
\Omega_{j(k)}^{2}\left(t_{0}\right)=0 \quad \text { for } \quad k \geq 1,
\end{gathered}
$$


then (91) reduces to the following set of equations

$$
\begin{gathered}
S_{j(1)}(t)=\left[\phi_{1}^{2}(t)-\phi_{1}^{2}\left(t_{0}\right)\right]+\frac{1}{2}\left[\Omega_{j(0)}^{2}(t)-\Omega_{j(0)}^{2}\left(t_{0}\right)\right], \\
S_{j(2)}(t)=\frac{1}{2} \Omega_{j(1)}^{2}(t)+\delta \lambda \cdot S_{j(1)}(t), \\
S_{j(3)}(t)=\frac{1}{2} \Omega_{j(2)}^{2}(t)+\delta \lambda \cdot S_{j(2)}(t), \ldots
\end{gathered}
$$

i.e. in the large- $N$ approximation we need only to calculate in the second order to identify the counterterms [18].

The renormalized vacuum mean field equation takes the form

$$
\ddot{\phi}_{1 j}+\left(m_{R}^{2}+S_{j}(t)\right) \phi_{1 j}=0 \text {. }
$$

Expanding the amplitudes $\tilde{W}_{\alpha j}$ and the dynamical phases $\tilde{\Theta}_{\alpha j}$ in power series in $\lambda_{R}$, it is also possible to deduce from Eqs.(75) and (78)-(79) a renormalized kinetic equation in a fixed order of perturbations as well as to develop a perturbation theory technique for the particle production problem. This work is in progress and will be reported elsewhere.

\section{Discussion}

1. For the model of a self-interacting complex $N$-component scalar field, we have derived in the large$N$ approximation a quantum kinetic equation with a non-Markovian source term. This equation determines the momentum distribution of the standard, non-tachyonic modes produced in quantum fluctuations of the scalar field around its vacuum mean value.

Our equation is complete in the sense that it includes all orders of the back-reaction and collision effects. Its form is compact and appropriate for the numerical study. We have obtained an explicit expression for the kinetic amplitudes related to collisions. These amplitudes account for the change in the particle number density in collisions in both "energy transition" and "collision" regimes.

Our method of derivation of kinetic equations is not restricted to the model discussed. It can be applied to any self-interacting field theory, providing the approximation scheme to treat non-diagonal interactions is specified. We have shown that in the large- $N$ approximation the functions $\Delta_{\alpha j}^{(1)}, \Delta_{\alpha j}^{(2)}$ vanish, so that the source term with higher order effects included has the same non-Markovian structure as the one with higher order effects omitted. However, beyond the large- $N$ approximation, the functions $\Delta_{\alpha j}^{(1)}, \Delta_{\alpha j}^{(2)}$ are expected to modify the structure of the source term as well.

2. For the particle production induced by an external electric field, the non-Markovian effects are known to be important when the field is strong [3]. For weak fields, non-Markovian effects disappear, and the Markovian limit of the kinetic equation, which is defined by the neglect of memory effects in the source term, can be successfully employed. If the external field is switched off, then the particle production stops.

For the self-interacting fields, the situation is completely different. The self-interaction takes place at all times and at all values of fields. It introduces memory effects, which appear even at lower orders of perturbations and can not be therefore neglected in any sensible limit or regime. Once the self-interaction is taken into consideration, the non-Markovian character of the source term is unavoidable. Even if the transition of energy from the background fields to fluctuations stops, the particle production process continues, being influenced essentially by the background field evolution history. 


\section{References}

[1] J. Rau and B. Müller, Phys. Repts. 272 (1996) 1.

[2] Y. Kluger, E. Mottola, and J.M. Eisenberg, Phys. Rev. D 58 (1998) 125015.

[3] S. Schmidt et al., Int. J. Mod. Phys. E 7 (1998) 709; Phys. Rev. D 59 (1999) 094005; J.C.R. Bloch et al., Phys. Rev. D 60 (1999) 116011; C.D. Roberts and S.M. Schmidt, Progr. Part. Nucl. Phys. 45 (2000) pp. S1-S103.

[4] C. Greiner, K. Wagner, and P.-G. Reinhard, Phys. Rev. C 49 (1994) 1693; H. Heiselberg and X. Wang, Phys. Rev. C 53 (1996) 1892; T.S. Biro and C. Greiner, Phys. Rev. Lett. 79 (1997) 3138; W.M. Alberico, A. Lavagno, and P. Quarati, Eur. Phys. J. C 12 (2000) 499; O.V. Utyuzh, G. Wilk, and Z. Wlodarczyk, J. Phys. G 26 (2000) L39.

[5] S. Ayik, M. Belkacem, and A. Bonasera, Phys. Rev. C 51 (1995) 611; S. Ayik et al., Phys. Rev. C 58 (1998) 1594.

[6] M. Colonna, M. Di Toro, and A. Guarnera, Nucl. Phys. A 580 (1994) 312.

[7] U. Fuhrmann, K. Morawetz, and R.Walke, Phys. Rev. C 58 (1998) 1473.

[8] K. Geiger, Phys. Rev. D 54 (1996) 949; D 56 (1996) 2665; S. Mrowczynski, Fiz. Elem. Chast. At. Yadra 30 (1999) 954 [ Phys. Part. Nuclei 30 (1999) 419 ]; S.A. Bass et al., Progr. Part. Nucl. Phys. 41 (1998) 225.

[9] D. Bodeker, Nucl. Phys. B 566 (2000) 402; P. Arnold, D.T. Son, and L.G. Yaffe, Phys. Rev. D 59 (1999) 105020; J.-P. Blaizot and E. Iancu, Nucl. Phys. B 557 (1999) 183; B 570 (2000) 326; D.F. Litim and C. Manuel, Nucl. Phys. B 562 (1999) 237.

[10] D. Boyanovsky, I.D. Lawrie, and D.-S. Lee, Phys. Rev. D 54 (1996) 4013; I.D. Lawrie and D.B. McKernan, Phys. Rev. D 55 (1997) 2290; F.T. Brandt, J. Frenkel, and A. Guerra, Int. J. Mod. Phys. A 13 (1998) 4281.

[11] D. Boyanovsky, H.J. de Vega, and S.-Y. Wang, Phys. Rev. D 61 (2000) 065006.

[12] D.B. Blaschke et al., Phys. Rev. D 65 (2002) 054039.

[13] F.M. Saradzhev, Phys. Lett. B 558 (2003) 103.

[14] J. Kapusta, Finite Temperature Field Theory (Cambridge Monographs on Mathematical Physics, 1989).

[15] J. Cornwall, R. Jackiw, and E. Tomboulis, Phys. Rev. D 10 (1974) 2428; S. Coleman, R. Jackiw, and H.D. Politzer, Phys. Rev. D 10 (1974) 2491; D.J. Gross and A. Neveu, Phys. Rev. D 10 (1975) 3235; F. Cooper, G.S. Guralnik, and S.H. Kasdan, Phys. Rev. D 14 (1976) 1607.

[16] T. Barnes and G. Ghandour, Phys. Rev. D 22 (1980) 924; W.A. Bardeen and M. Moshe, Phys. Rev. D 28 (1983) 1372; C. Bender and F. Cooper, Ann. Phys. (N.Y.) 160 (1985) 323.

[17] G.F. Mazenko, Phys. Rev. Lett. 54 (1985) 2163; Phys. Rev. D 34 (1986) 2223.

[18] F. Cooper and E. Mottola, Phys. Rev. D 36 (1987) 3114; Phys. Rev. D 40 (1989) 456. 